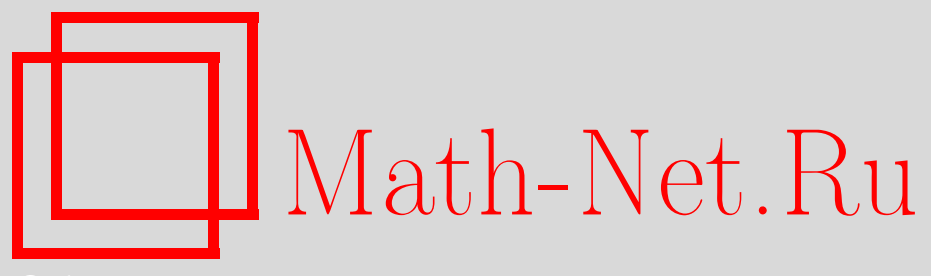

М. Мехрафарин, Геометрический подход к канонической переформулировке квантовой механики, ТMФ, 2006, том 147, номер 3, 479-486

DOI: https://doi.org/10.4213/tmf1989

Использование Общероссийского математического портала Math-Net.Ru подразумевает, что вы прочитали и согласны с пользовательским соглашением http://www.mathnet.ru/rus/agreement

Параметры загрузки:

IP : 54.198 .67 .100

26 апреля 2023 г., 07:30:37

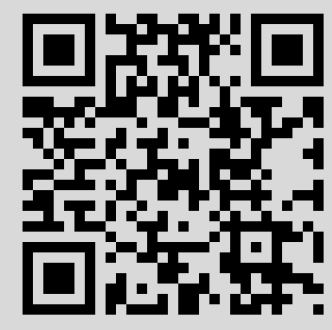




\title{
ГЕОМЕТРИЧЕСКИЙ ПОДХОД К КАНОНИЧЕСКОЙ ПЕРЕФОРМУЛИРОВКЕ КВАНТОВОЙ МЕХАНИКИ
}

\begin{abstract}
Мера различимости двух соседних приготовлений физической системы с помощью измерительного прибора естественным образом определяет элемент длины на пространстве приготовлений данной системы. Показано, что квантовую механику можно вывести из инвариантности этого элемента длины. Обсуждается также каноническая формулировка квантовой статистической механики.
\end{abstract}

Ключевые слова: приготовление, метрика различимости, измерительный прибор, каноническая квантовая механика.

\section{1. ВВЕДЕНИЕ}

Квантово-механическое описание имеет дело с дополнительными контекстами, задаваемыми несовместными измерениями, и именно по отношению к такому контексту описываются "приготовления" (например, приготовленные состояния физической системы). Такая контекстуальность описания проявляется в стандартной формулировке квантовой механики: различные базисы (представления) в гильбертовом пространстве задаются несовместными измерениями через собственные состояния $\{|i\rangle\}$ измеряемой наблюдаемой, где $i=1, \ldots, n$ нумерует результаты измерения, а изменение базиса (представления), таким образом, соответствует изменению контекста измерения. Вектор состояния $|\psi\rangle$, соответствующий приготовлению, описывается по отношению к такому базису с помощью комплексных компонент $\psi_{i}=\sqrt{p_{i}} e^{i \phi_{i}}$, где $\left\{p_{i}\right\}$ - распределение вероятностей результатов измерений, а $\phi_{i}-$ квантовая фаза приготовления, соответствующего результату $i$. Поэтому совокупность (вещественных) значений $\left(p_{i}, \phi_{i}\right)$ описывает произвольное приготовление по отношению к данному измерительному контексту (измерительному прибору). Совокупность всех приготовлений можно назвать пространством приготовлений системы, а произвольное приготовление представить точкой в этом пространстве с координатами $\left(p_{i}, \phi_{i}\right)$ по отношению к данному измерительному контексту. Измерительные приборы (контексты), таким образом, задают системы отсчета или системы координат в пространстве приготовлений, в которых описывается точка (приготовление),

${ }^{*}$ Physics Department, Amirkabir University of Technology, Tehran 15914, Iran. E-mail: mehrafar@aut.ac.ir 
с распределениями вероятностей результатов измерения и соответствующими фазами в качестве ее координат. Заметим, однако, что, несмотря на контекстуальность квантово-механического описания, каждая система отсчета (измерительный контекст) эквивалентна каждой другой системе (контексту) в том, что касается описания; выделенного измерения нет.

Итак, имеется естественная мера того, чем различаются между собой два вектора состояния (приготовления) в гильбертовом пространстве, а именно угол между соответствующими лучами. Для двух соседних приготовлений имеем

$\left[\cos ^{-1}|\langle\psi \mid \psi+d \psi\rangle|\right]^{2}=\sum_{i} \frac{d p_{i}^{2}}{4 p_{i}}+\sum_{i} p_{i} d \phi_{i}^{2}-\left(\sum_{i} p_{i} d \phi_{i}\right)^{2}+$ члены высших порядков.

Поскольку любые два приготовления в пространстве приготовлений, естественно, различаются расстояниями, указанный угол определяет (риманов) элемент длины на пространстве приготовлений согласно формуле

$$
d s^{2}=\sum_{i} \frac{d p_{i}^{2}}{4 p_{i}}+\sum_{i} p_{i} d \phi_{i}^{2}-\left(\sum_{i} p_{i} d \phi_{i}\right)^{2}
$$

Этот элемент длины (также известный как метрика Фубини-Штуди [1]) представляет собой меру различимости двух соседних приготовлений $\left(p_{i}, \phi_{i}\right)$ и $\left(p_{i}+d p_{i}, \phi_{i}+d \phi_{i}\right)$ посредством данного измерительного прибора. Однако, поскольку все измерительные контексты эквивалентны в смысле описания заданного приготовления и не имеется выделенного предпочтительного измерения, мера различимости состояний должна быть инвариантна по отношению ко всем измерительным приборам (в противном случае некоторые измерения оказались бы более различающими, чем другие, что создало бы основу для предпочтений).

В разделе 2 в рамках подхода, определяемого пространством приготовлений, мы покажем, что квантовую механику можно вывести из инвариантности элемента длины в канонической формулировке. Первое слагаемое в выражении для элемента длины представляет собой хорошо известную меру различимости двух соседних распределений вероятности [2], а второе слагаемое - дисперсию разности фаз. Хотя каноническая формулировка хорошо известна [3], поставленный нами акцент на фундаментальном значении, имеющем элемент длины, придает значительную глубину пониманию основ квантовой механики при условии, что элемент длины можно получить из независимых предпосылок. Такие предпосылки, из которых следует, что элемент длины представляет собой сумму двух указанных выше слагаемых, даны в работе [4]. Наконец, в разделе 3 мы обсуждаем каноническую формулировку квантовой статистической механики в пространстве приготовлений.

\section{2. КАНОНИЧЕСКАЯ КВАНТОВАЯ МЕХАНИКА}

В этом разделе мы дадим краткий обзор основ канонической квантовой теории, делая акцент на роли инвариантности элемента длины (1). Инвариантность элемента длины ограничивает вид возможных координатных преобразований $\left(p_{i}, \phi_{i}\right) \rightarrow$ 
$\left(p_{i}^{\prime}, \phi_{i}^{\prime}\right)$ в пространстве приготовлений. Такие преобразования определяют, как данное приготовление должно описываться относительно различных измерительных контекстов. Нам не требуется выводить законы преобразования с нуля. Вигнер показал [5], что наиболее общим сохраняющим углы преобразованием в гильбертовом пространстве является унитарное преобразование. Поэтому искомые преобразования в пространстве приготовлений соответствуют унитарным преобразованиям в стандартной формулировке, связанным с изменением базиса. Записывая матрицу унитарного преобразования в виде $u_{j i}=\sqrt{\omega_{i j}} e^{i \beta_{i j}}$, для унитарного преобразования $\psi_{i}^{\prime}=\sum_{j} u_{j i}^{*} \psi_{j}$ получим

$$
\begin{aligned}
p_{i}^{\prime} & =\sum_{j k} \sqrt{\omega_{i j} p_{j}} \sqrt{\omega_{i k} p_{k}} \cos \left(\phi_{j k}-\beta_{i j}+\beta_{i k}\right), \\
\operatorname{tg} \phi_{i}^{\prime} & =\frac{\sum_{j} \sqrt{\omega_{i j} p_{j}} \sin \left(\phi_{j}-\beta_{i j}\right)}{\sum_{j} \sqrt{\omega_{i j} p_{j}} \cos \left(\phi_{j}-\beta_{i j}\right)},
\end{aligned}
$$

где $\phi_{j k}=\phi_{j}-\phi_{k}$ - относительная фаза, а $2 n^{2}$ параметров преобразования $\omega_{i j}$ и $\beta_{i j}$ удовлетворяют соотношениям

$$
\begin{aligned}
\sum_{i}{\sqrt{\omega_{i j} \omega_{i k}}}_{\sin }^{\cos }\left(\beta_{i k}-\beta_{i j}\right) & =\sum_{i}{\sqrt{\omega_{j i} \omega_{k i}}}_{\sin }^{\cos }\left(\beta_{k i}-\beta_{j i}\right)=0, \quad j \neq k, \\
\sum_{i} \omega_{i j} & =\sum_{i} \omega_{j i}=1 .
\end{aligned}
$$

Имеется $n^{2}$ связей, которые выражают условия унитарности $\sum_{i} u_{i j}^{*} u_{i k}=\sum_{i} u_{j i} u_{k i}^{*}=$ $\delta_{j k}$, оставляя независимыми только $n^{2}$ параметров преобразования.

Преобразование координат (2) представляет собой требуемый закон преобразования в пространстве приготовлений, который в терминах $n^{2}$ независимых параметров связывает описания заданного приготовления по отношению к различным измерительным контекстам. Далее мы покажем, что закон эволюции отдельного приготовления (уравнение Шредингера) также естественным образом следует из того же свойства инвариантности.

Рассмотрим в пространстве приготовлений изолированной системы произвольное приготовление, заданное координатами $\left(p_{i}, \phi_{i}\right)$ по отношению к данному измерительному прибору. Поскольку нет предпочтительной системы отсчета, уравнения, которым подчиняется развитие приготовления во времени, должны быть ковариантными относительно закона преобразования (2). После некоторых преобразований с использованием соотношений (3) из уравнения (2) следует, что

$$
M J M^{\mathrm{T}}=J,
$$

где $(2 n \times 2 n)$-матрицы $M$ и $J$ имеют вид

$$
M=\left(\begin{array}{ll}
\frac{\partial p_{i}^{\prime}}{\partial p_{j}} & \frac{\partial p_{i}^{\prime}}{\partial \phi_{j}} \\
\frac{\partial \phi_{i}^{\prime}}{\partial p_{j}} & \frac{\partial \phi_{i}^{\prime}}{\partial \phi_{j}}
\end{array}\right), \quad J=\left(\begin{array}{cc}
0 & \delta_{i j} \\
-\delta_{i j} & 0
\end{array}\right)
$$

6 Теоретическая и математическая физика, т. 147, № 3, 2006 г. 
Видно, что уравнение (4) выражает необходимое и достаточное условие (условие симплектичности; см., например, [6]) каноничности координатного преобразования (2), т.е. необходимое и достаточное условие ковариантности уравнений типа уравнений Гамильтона

$$
\dot{p}_{i}=\frac{\partial \mathcal{H}}{\partial \phi_{i}}, \quad \dot{\phi}_{i}=-\frac{\partial \mathcal{H}}{\partial p_{i}}
$$

относительно этого преобразования, где $\mathcal{H}$ - скаляр $\left(\mathcal{H}\left(p_{i}, \phi_{i}, t\right)=\mathcal{H}^{\prime}\left(p_{i}^{\prime}, \phi_{i}^{\prime}, t\right)\right)$, имеющий, конечно, размерность $\left[\right.$ время $\left.^{-1}\right]$. Если принять $\hbar=1$, то понятно, что гамильтониан $\mathcal{H}$ должен быть отождествлен со средней (ожидаемой) энергией приготовления, которая имеет одно и то же значение во всех системах отсчета (представлениях). Тогда канонические уравнения (6), являющиеся единственной системой уравнений, ковариантных относительно преобразований (2), выступают в качестве единственного кандидата на роль “уравнений движения" приготовления. Чтобы установить связь со стандартной формулировкой квантовой механики, запишем

$$
\mathcal{H}\left(p_{i}, \phi_{i}, t\right)=\langle\psi|H| \psi\rangle=\sum_{i j} H_{i j}(t) \sqrt{p_{i} p_{j}} e^{-i \phi_{i j}},
$$

где $H$ - оператор Гамильтона (возможная временна́я зависимость которого приводит к явной временно́й зависимости гамильтониана $\mathcal{H}$ ). Таким образом, уравнения (6) переходят в уравнение $i \dot{\psi}_{i}=\sum_{j} H_{i j} \psi_{j}$, которое является не чем иным, как уравнением Шредингера в представлении, задаваемом измерительным прибором; ковариантность уравнений (6) относительно преобразования (2) соответствует ковариантности последнего относительно унитарных преобразований.

Нет необходимости подчеркивать, что и группа унитарного преобразования в квантовой механике, и уравнение Шредингера возникли из свойства инвариантности пространства приготовлений.

Таким образом, динамика в пространстве приготовлений сильно напоминает классическую динамику, описываемую в фазовом пространстве. В частности, канонически сопряженные координаты $\left(p_{i}, \phi_{i}\right)$ определяют эволюционную траекторию приготовления в пространстве приготовлений изолированной системы со средней энергией $\mathcal{H}$. Более того, из-за развития приготовления во времени среднее значение произвольной наблюдаемой $F$, а именно скаляр

$$
f\left(p_{i}, \phi_{i}, t\right) \equiv\langle\psi|F| \psi\rangle=\sum_{i j} F_{i j}(t) \sqrt{p_{i} p_{j}} e^{-i \phi_{i j}},
$$

становится динамической переменной в пространстве приготовлений. Его динамика следует из уравнений движения (6), определяемых из уравнения

$$
\dot{f}=\frac{\partial f}{\partial t}+\sum_{i}\left(\frac{\partial f}{\partial p_{i}} \frac{\partial \mathcal{H}}{\partial \phi_{i}}-\frac{\partial f}{\partial \phi_{i}} \frac{\partial \mathcal{H}}{\partial p_{i}}\right) \equiv \frac{\partial f}{\partial t}+\{f, \mathcal{H}\},
$$

где $\{f, \mathcal{H}\}$ - скобки Пуассона для $f$ и $\mathcal{H}$. Нет необходимости говорить, что, поскольку скобки Пуассона инвариантны относительно канонических преобразований, динамика не зависит от выбора системы отсчета измерительного прибора. Уравнение (7), 
разумеется, соответствует уравнению $\dot{f}=\langle\dot{F}\rangle+(1 / i)\langle[F, H]\rangle$ в стандартной формулировке квантовой механики, поскольку, как можно непосредственно показать,

$$
\langle\dot{F}\rangle=\sum_{i j} \dot{F}_{i j} \sqrt{p_{i} p_{j}} e^{-i \phi_{i j}}=\frac{\partial f}{\partial t}
$$

$$
\frac{1}{i}\langle[F, H]\rangle=\{\langle F\rangle,\langle H\rangle\}=\{f, \mathcal{H}\} .
$$

\section{3. КАНОНИЧЕСКАЯ КВАНТОВАЯ СТАТИСТИЧЕСКАЯ МЕХАНИКА}

Точка в пространстве приготовлений соответствует чистому состоянию, т.е. максимальной информации о системе на заданный момент времени, совместной с объективными данными, полученными из измерений во всех возможных экспериментах с наблюдаемыми системы. В идеале, когда такая предварительная информация доступна в качестве начальных данных, последующая временна́я эволюция состояния определяется уравнениями движения типа уравнений Гамильтона. В противном случае развитие во времени представляющих интерес физических величин следует выводить из неполной информации. Мы покажем, что канонический подход приводит к переформулировке квантовой статистической механики, аналогичной формулировке классической статистической механики в фазовом пространстве.

Используя каноническую формулировку, по аналогии с классической механикой в фазовом пространстве временну́ю эволюцию изолированного приготовления можно представить в виде последовательности инфинитезимальных канонических преобразований, порожденных $\mathcal{H}$. Тогда, поскольку при канонических преобразованиях

$$
\delta\left(\sum_{i} p_{i}^{\prime}-1\right) d^{n} p^{\prime} d^{n} \phi^{\prime}=\delta\left(\sum_{i} p_{i}-1\right)\|M\| d^{n} p d^{n} \phi=\delta\left(\sum_{i} p_{i}-1\right) d^{n} p d^{n} \phi,
$$

элемент объема $d \mu=\delta\left(\sum_{i} p_{i}-1\right) d^{n} p d^{n} \phi$ пространства приготовлений остается инвариантным во времени. Отсюда следует, что распределение вероятностей точек $w\left(p_{i}, \phi_{i}, t\right)$ в пространстве приготовлений также является константой движения, т.е.

$$
\dot{w}=\frac{\partial w}{\partial t}+\{w, \mathcal{H}\}=0 .
$$

Это уравнение, являющееся уравнением типа уравнения Лиувилля, актуально, когда максимальная информация, позволяющая определить приготовление однозначно, недоступна, и поэтому приходится иметь дело с вероятностным распределением приготовлений (смешанный ансамбль), совместным с заданной информацией. Предположим, что предварительная информация состоит из экспериментальных результатов измерений некоторой наблюдаемой $F$ системы, стандартным образом выражаемой в терминах ее среднего значения $\bar{F}$ при $t=0$ (обобщение на некоторое число коммутирующих наблюдаемых выполняется легко). Информационная энтропия, связанная с этим измерением, равна

$$
S=-\sum_{i=1}^{n} \rho_{i} \ln \rho_{i}
$$


где $\rho_{i}$ - вероятность получения собственного значения $F_{i}$ наблюдаемой $F$. Вероятности $\left\{\rho_{i}\right\}$, которые лучше всего описывают информацию, получаются путем максимизации энтропии при заданном результате измерения

$$
\sum_{i} \rho_{i} F_{i}=\bar{F}
$$

и условии нормировки

$$
\sum_{i} \rho_{i}=1
$$

В результате получаем

$$
\rho_{i}=\frac{e^{-\beta F_{i}}}{Z}
$$

где $Z=\sum_{i} e^{-\beta F_{i}}-$ статистическая сумма, а $\beta$ определяется из условия $(10)$, которое можно теперь записать в виде

$$
-\frac{\partial}{\partial \beta} \ln Z=\bar{F}
$$

Относительно рассматриваемого нами измерительного контекста произвольное приготовление в ансамбле имеет координаты $\left(p_{i}, \phi_{i}\right)$, где $p_{i}$ - вероятность, соответствующая результату $F_{i}$. Поэтому, беря в качестве весов соответствующие вероятности $w$ приготовлений, получаем

$$
\rho_{i}=\int w p_{i} d \mu
$$

где интеграл берется по всему пространству $\left(0 \leqslant p_{i} \leqslant 1, \quad 0 \leqslant \phi_{i} \leqslant 2 \pi\right)$. Таким образом, связи (10) и (11) переходят в связи на $w$ согласно соотношениям

$$
\begin{gathered}
\int w f d \mu=\bar{F}, \\
\int w d \mu=1,
\end{gathered}
$$

соответственно $\left(f=\langle F\rangle=\sum_{i} p_{i} F_{i}\right)$. Их каноническая инвариантность становится явной $\left(w, f\right.$ и $d \mu$ суть скаляры). Когда $\rho_{i}$ задается уравнением $(12)$, интегральное уравнение (14) можно решить относительно $w$, что дает

$$
w_{0}\left(p_{i}, \phi_{i}\right)=(n+1) \frac{\left\langle e^{-\beta F}\right\rangle}{\int\left\langle e^{-\beta F}\right\rangle d \mu}-\frac{n !}{(2 \pi)^{n}} .
$$

Условие (15), которое определяет $\beta$, можно теперь записать в виде

$$
-\frac{\partial}{\partial \beta} \ln \int\left\langle e^{-\beta F}\right\rangle d \mu=\bar{F} .
$$

Это уравнение представляет собой, конечно же, как раз уравнение (13), поскольку

$$
\int\left\langle e^{-\beta F}\right\rangle d \mu=\sum_{i} e^{-\beta F_{i}} \int p_{i} d \mu=\frac{(2 \pi)^{n}}{n !} Z .
$$


В проведенных выше вычислениях полезен следующий результат:

$$
\int_{0}^{1} p_{1}^{m_{1}} \ldots p_{n}^{m_{n}} \delta\left(\sum_{i} p_{i}-1\right) d^{n} p=\frac{\prod_{i}\left(m_{i} !\right)}{\left(\sum_{i} m_{i}+n-1\right) !} .
$$

Распределение (16) в пространстве приготовлений представляет собой результат измерения при $t=0$. Его можно использовать в качестве начального значения для уравнений типа уравнения Лиувилля (9), чтобы получить $w\left(p_{i}, \phi_{i}, t\right)$. Тогда ожидаемое значение любой наблюдаемой $Q$ в произвольный момент времени $t$ будет даваться уравнением

$$
\int w\left(p_{i}, \phi_{i}, t\right) q d \mu=\bar{Q}(t)
$$

где, конечно, $q\left(p_{i}, \phi_{i}\right)=\langle Q\rangle$ - соответствующая динамическая переменная в пространстве приготовлений. Для равновесных распределений $\partial_{t} w=0$, так что согласно уравнению $(9)\{w, \mathcal{H}\}=0$. Поскольку из соотношения (8) мы имеем

$$
\left\{\left\langle e^{-\beta F}\right\rangle, \mathcal{H}\right\}=\frac{1}{i}\left\langle\left[e^{-\beta F}, H\right]\right\rangle,
$$

то оказывается, что если $F$ является константой движения, то распределение, задаваемое соотношением (16), будет равновесным. В качестве примера можно немедленно привести каноническое распределение, для которого $F=H$.

Поучительно теперь провести прямое соответствие со стандартной формулировкой квантовой механики в терминах оператора плотности $\rho$. По определению по отношению к произвольному базису измерений имеет место соотношение

$$
\rho_{i j}(t)=\int w \psi_{i} \psi_{j}^{*} d \mu=\int w\left(p_{i}, \phi_{i}, t\right) \sqrt{p_{i} p_{j}} e^{i \phi_{i j}} d \mu .
$$

Отсюда следует, что

$$
\begin{gathered}
\operatorname{tr} \rho=\sum_{i} \rho_{i i}=\int w d \mu, \\
\operatorname{tr}(\rho F)=\sum_{i j} \rho_{i j} F_{j i}=\int w f d \mu .
\end{gathered}
$$

Условие нормировки и формула для средних, таким образом, редуцируются к виду, привычному для стандартной формулировки. Более того, если система отсчета такова, что в функциональном виде выражения для $w$ отсутствуют фазы, то путем интегрирования по фазам выражение (17) редуцируется к выражению вида $\rho_{i j}=\rho_{i} \delta_{i j}$, где $\rho_{i}$ дается выражением (14). Это соответствует диагональному представлению для $\rho$ с собственными значениями $\left\{\rho_{i}\right\}$. Поэтому энтропия также сводится к привычному выражению $S=-\operatorname{tr}(\rho \ln \rho)$. Окончательно для построения полного соответствия мы должны доказать эквивалентность уравнения фон Неймана для $\rho_{i j}$ и уравнения типа уравнения Лиувилля для $w$. Один подход заключается в том, чтобы подставить выражение (17) в уравнение фон Неймана и получить уравнения типа уравнения Лиувилля (9) как необходимое и достаточное условие. Однако 
более простое доказательство получается, если заметить, что в силу

$$
(2 \pi)^{n} w=(n+1) !\langle\rho\rangle-n !
$$

(что легче всего вывести из выражения (17) в диагональном представлении) мы имеем

$$
\dot{w}=\frac{(n+1) !}{(2 \pi)^{n}}\left\langle\dot{\rho}+\frac{1}{i}[\rho, H]\right\rangle .
$$

Поэтому из уравнения фон Неймана следует, что $\dot{w}=0$ (и обратно), поскольку среднее берется по произвольному приготовлению.

Как мы показали, в канонической квантовой статистической механике распределение вероятностей приготовлений и уравнение типа уравнения Лиувилля для него заменяют оператор плотности и уравнение фон Неймана из стандартной формулировки. Каноническая переформулировка сильно похожа на классическую статистическую механику, за исключением выражения для энтропии, а именно,

$$
S=-\int w\langle\ln \rho\rangle d \mu,
$$

из-за существования квантовых вероятностей $p_{i}\left(S_{\text {класс }}=-\int w \ln w d \mu\right)$.

\section{Список литературы}

[1] J. Anandan, Y. Aharanov, Phys. Rev. Lett., 65 (1990), 1697; J. Anandan, Found. Phys., 21 (1991), 1265; G. W. Gibbons, J. Geom. Phys., 8 (1992), 147; S. L. Braunstein, C. M. Caves, Phys. Rev. Lett., 72 (1994), 3439.

[2] R. A. Fisher, Proc. Roy. Soc. Edinburgh, 42 (1922), 321; A. Bhattacharyya, Bull. Calcutta Math. Soc., 35 (1943), 99; W.K. Wootters, Phys. Rev. D, 23 (1981), 357.

[3] A. Ashtekar, T.A. Schilling, Geometrical formulation of quantum mechanics, gr-qc/9706069; S. Weinberg, Ann. Phys., 194 (1989), 336; M. J. W. Hall, M. Reginatto, J. Phys. A, 35 (2002), 3289; M. J.W. Hall, K. Kumar, M. Reginatto, J. Phys. A, 36 (2003), 9779; N.P. Landsman, Mathematical Topics Between Classical and Quantum Mechanics, Springer, New York, 1998; F. Guerra, R. Marra, Phys. Rev. D, 29 (1984), 1647; D. Minic, C. H. Tze, Phys. Lett. B, 536 (2002), 305; 581 (2004), 111; Phys. Rev. D, 68 (2003), 061501.

[4] M. Mehrafarin, Int. J. Theor. Phys., 44 (2005), 429.

[5] Е. Вигнер, Теория групn, ИЛ, М., 1961.

[6] Г. Голдстейн, Классическая механика, Наука, М., 1974.

Поступила в редакцию 21.XI.2005 г. 